\title{
FINITE ELEMENT STUDY OF THE ACCOMMODATION BEHAVIOUR OF THE CRYSTALLINE LENS AFTER fS-LASER TREATMENT
}

\author{
Besdo $\mathrm{S}^{1}$, Wiegand $\mathrm{J}^{1}$, Hahn $\mathrm{J}^{2}$, Ripken $\mathrm{T}^{2}$, Krüger $\mathrm{A}^{2}$, Fromm $\mathrm{M}^{3}$, Lubatschowski $\mathrm{H}^{3}$ \\ ${ }^{1}$ Institute for Continuum Mechanics, Leibniz Universität Hannover, Germany \\ ${ }^{2}$ Laser Zentrum Hannover, Germany \\ ${ }^{3}$ Rowiak GmbH, Germany \\ s.besdo@ikm.uni-hannover.de
}

\begin{abstract}
With aging the ability of the crystalline lens to adapt to different viewing distances decreases. Until now there is no satisfying treatment available. It is possible to influence the deformability of the crystalline lens by inducing cuts using femtosecond ( $f$ s)-laser. To test the influence of several different cutting geometries is expensive and a huge amount of crystalline lenses is needed. Finit Element Method offers the possibility to test the influence of different cutting geometries on the flexibility. Therefore, it is necessary to develop a method to simulate the cuts in an adequate way. In first simulations cuts were assumed to be isotropic inter layers. Later on, effective material properties for the inter layer were calculated with a homogenization technique. The results confirm the influence of the cuts on the flexibility.
\end{abstract}

Keywords: crystalline lens, fs-Laser, lentotomiy, presbyopia

\section{Introduction}

The ability of the crystalline lens to focus on different viewing distances decreases with aging. In most cases it is presbyopia, but the reason for the phenomena is not well understood so far. One explanation is that the cells under the capsule grow during the whole life. Thus the amount of material within the lens capsule increases with time. As a result the flexibility of the lens decreases and the lens becomes stiffer. Until now there is no satisfying treatment. The most common treatment is the use of eyeglasses. Laser-treatments offer new possibilities. One already established method for the treatment of ametropia is the Lasik surgery. The curvature of the cornea is changed during a surgery by the use of a laser.

Another approach seems to be to induce cuts with a femtosecond (fs) laser into the crystalline lens to influence the accommodation behaviour. First experiments showed promising results. In vitro tests on eye-lenses of different species showed that the lens thickness and the curvature of the lens change after fs-laser treatment during simulated accommodation [1]. The testing of different cutting geometries is relatively expensive and a huge amount of crystalline lenses is needed. Human and primate crystalline lenses are rare and expensive. Another problem is the short time period between death and the changes in the behaviour of the lens. Therefore a Finite Element (FE) model was developed to be able to simulate the influence of fs-laser cuts on the accommodation behaviour.

\section{Methods}

A Finite element model of a 45 years old donor was generated on the basis of literature [2]. Within the capsule the lens has areas with different stiffness values which increase with aging. In young people the inner part (nucleus) has the same or softer material parameters than the surrounding material (cortex) (Fig. 1).

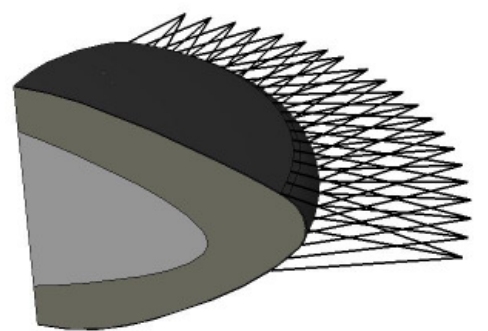

Figure 1: Model of a human crystalline lens with nucleus (light grey), cortex (grey), capsule (dark grey) and zonules (black)

During aging the nucleus gets stiffer than the cortex. The material properties used in this study are taken from Burd et al. [3], see Table 1.

Table 1: Young's moduli for the crystalline lenses of a 45 years old donor by Burd [3] in N/mm²

\begin{tabular}{cc}
\hline & 45 \\
\hline Nucleus & 0.0009966 \\
Cortex & 0.00398 \\
Capsule & 1.45 \\
\hline
\end{tabular}

As a first cutting geometry a so called steering wheel was chosen with 12 segments over the whole circumference (see Figure 2).

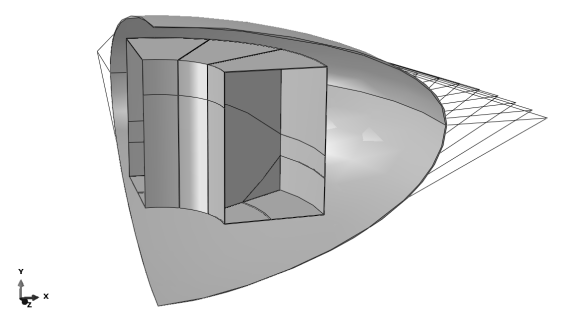

Figure 2: Model of a quarter of the capsule and the 'steeringwheel' cutting geometry 
The laser focus points are $5 \mu \mathrm{m}$ in diameter and the distance between two focus points is $5 \mu \mathrm{m}$, too. All simulations were performed with ABAQUS $®$ from Dassault Systèmes using quadratic tetrahedrons. In a first approach the cutting areas were simulated by an inter layer with isotropic material properties. The Young's modulus was assumed to be $1 \cdot 10^{-6} \mathrm{~N} / \mathrm{mm}^{2}$ and a Poisson ratio of 0.49 was set.

In a second step effective material properties were calculated for the inter layer in the nucleus and in the cortex using a homogenisation method by Hain et al. [4]. Therefore, a representative reference volume (RV) was modelled as shown in Figure 3. The RV is embedded into a so called window. The wall thickness of the window was $50 \%$ of the RV.

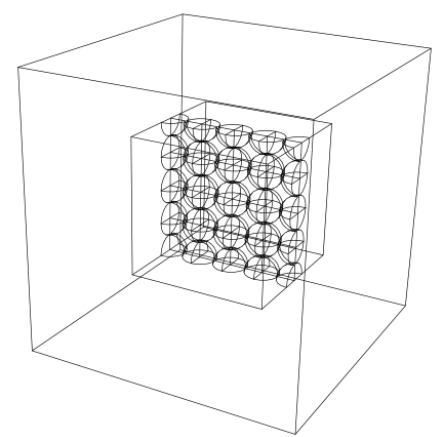

Figure 3: Embedded representative reference volume for the inter layer

Within an iteration process the material properties of the surrounding material were adapted till its behaviour was similar to the behaviour of the RV. To calculate the 36 material constants three tensile tests and three shear tests were simulated. The strains for defined loadings were calculated and the new material properties for surrounding area were determined for the next iteration step, until an abort criterion was reached. The new anisotropic material properties were used for the inter layer taking the orientation of the inter layer into account.

In the eye the zonules carry the contractions of the ciliary muscle to the lens. Within the model the zonules were represented by 21 bundles of three beams representing the anterior, central and posterior zonule, respectively. The zonules were connected to the capsule via multi point constrains. To simulate the stretching of the lens each crossing point of three beams was moved $0.2767 \mathrm{~mm}$ in radial direction.

The lens thickness after stretching was used to assess the results.

\section{Results}

The diameter of the lens before cutting changed from $8.896 \mathrm{~mm}$ to $9.376 \mathrm{~mm}$ at the lens equator, the thickness of the lens changed $0.484 \mathrm{~mm}$ from $4.134 \mathrm{~mm}$ to $3.649 \mathrm{~mm}$.

With isotropic material parameters for the inter layer the change in the diameter is $0.480 \mathrm{~mm}$ as well. The thickness becomes $0.496 \mathrm{~mm}$ thinner in the stretched position compared to the un-stretched position. The results for the model with effective material properties show a difference in the diameter of $0.480 \mathrm{~mm}$ and in the thickness of $0.518 \mathrm{~mm}$.

The results for the displacement of the lens after cutting simulated with anisotropic material properties are shown in Figure 4.

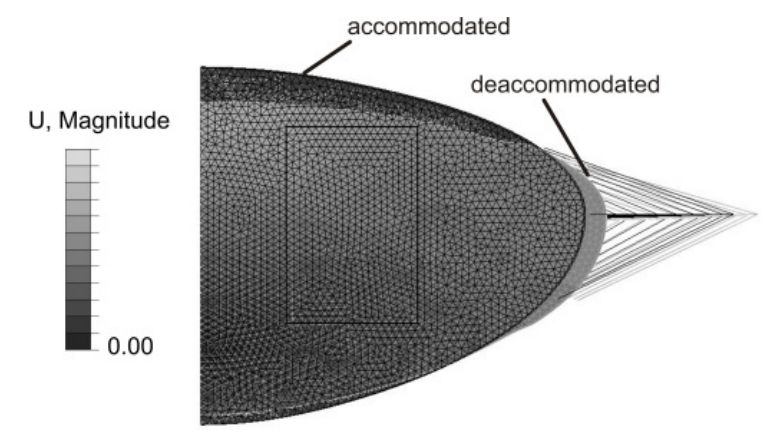

Figure 4: Displacements for a stretched crystalline lens after inducing cuts

\section{Discussion}

The influence of the material properties within the inter layer on the changes of the diameter is negligible. But the material properties have an influence on the thickness change. It was possible to show, that the flexibility of the lens increases after cutting. This confirms experimental results with porcine crystalline lenses and crystalline lenses of primates. The results of the simulations with anisotropic material properties for the inter layer show a smaller influence on the changes in the thickness than the isotropic materials. In a next step primate lenses will be examined in vitro and Finite Element models will be generated of the same samples. A comparison of the simulated and the experimental data will show which assumption is more realistic. Both methods seem to be appropriate to test whether cutting geometries will have a positive influence on the lens flexibility.

\section{Acknowledgement}

Thanks to the Deutsche Forschungsgemeinschaft (DFG) for funding this project (BE3990-3).

\section{Bibliography}

[1] Krueger, R.R., Kuszak, J. et al.: First safety study of femtosecond laser photodisruption in animal lenses: Tissue morphology and cataractogenesis. J Cataract Refract Surgery, vol. 31, pp. 2386-2394, December 2005

[2] Hermanns, E.A., Dubbelmann, M., et al.: Estimating the external force scting on the human eye lens during accommodation by finite element modelling, Vision Research, vol. 46, pp. 3642-3650, October 2006

[3] Burd, H., Judge, S., Cross, J.: Numerical Modelling of the accommodating lens. Vision Research, vol. 42, pp. 2235-2251, August 2002

[4] Hain, M., Wriggers, P.: Numerical homogenization of hardened cement paste, Comput Mech, vol. 42, pp. 197212, July 2008 